\title{
To Understand the Modulatory Effect of Probiotics on Irritable Bowel Syndrome
}

TO THE EDITOR: "I wonder why my IBS symptoms start with every midterm test no apparent reason in food whatever it is in clean or not.... The only relief I've had is from probiotics such as yogurt and Saccharomvces Boulardii Sachets when I take them consistently." The complaint here is from a third year high school student whose recurrent irritable bowel syndrome (IBS) was interrelated between gut symptoms and fluctuation of ACTH $(18.55 \mu \mathrm{g} / \mathrm{dL}$ at $8 \mathrm{AM}$ and $7.71 \mu \mathrm{g} / \mathrm{dL}$ at $4 \mathrm{PM})$. Indeed, when he had diet prebiotics or took $S$. Boulardii Sachets in advance of a midterm test his symptoms could be attenuated and the level of ACTH could be back to normal. This similar consultancy comes extremely more frequent during the period of national (China) university admission exam in June.

Despite that it is very common to see IBS outpatients in adopting good diet and probiotics, emotion control, and medical care, we still cannot use knowledge from pathophysiology studies to IBS patients in a clinically meaningful way. In everyday clinical work, we still rely on subgrouping patients based on the predominant bowel habit when we decide how to manage and treat the patients. Recently, a paper was published in the Journal of Neurogastroenterol Motil entitled "Bifidobacterium longum and Lactobacillus helveticus synergistically suppress stress-related visceral hypersensitivity through hypothalamic-pituitary-adrenal axis modulation." ${ }^{1}$ This is a study extremely concerned to our understanding on how a dysregulated stress-gut-microbiome axis promotes IBS, and what therapeutic measures can be used to modulate this axis and therefore reverse the course of the disease.

Ait-Belgnaoui et $\mathrm{al}^{1}$ used the water avoidance stress (WAS) method creating an IBS mice model, under which the colorectal distension measurement was detected to represent IBS symptom. The effects of visceral perception and neuro-hormones, as well as expression of the glucocorticoid receptor, were examined by using probiotic formulation including both or single Lactobacillus helveticus R0052 and Bifidobacterium longum R0175. The results demonstrated that stress can cause visceral pain to a higher perception as seen from Figure 1, in which the chronic stress significantly exaggerates the amplitude of the perception, and such exaggerated symptoms can be significantly attenuated by feeding $B$. longum and/or $L$. helveticus. Furthermore, the authors uncover that stressor can significantly elevate the plasma stress hormones including corticosterone/catecholamine and down-regulate mRNA of glucocorticoid receptor gene expression among hypothalamus, hippocampus, and prefrontal cortex. Surprisingly, those abnormalities can also be treated by probiotic(s) toward a balanced level in laboratory. As seen from Figure 2 the plasma corticosterone $(\mathrm{ng} / \mathrm{mL})$ was 3 times higher in the WAS vehicle compared to that of control vehicle. While the probiotics of $B$. longum and $L$. helveticus can synergistically suppress corticosterone from the elevated higher level to a similar level as in the control vehicle, or lower down it remarkably when a single probiotic was used. Together, this study finds that chronic stress or depression can increase visceral pain and central nervous hormones imbalance. In addition, the authors find, in their previous study, that WAS can damage gut permeability (tight junc-

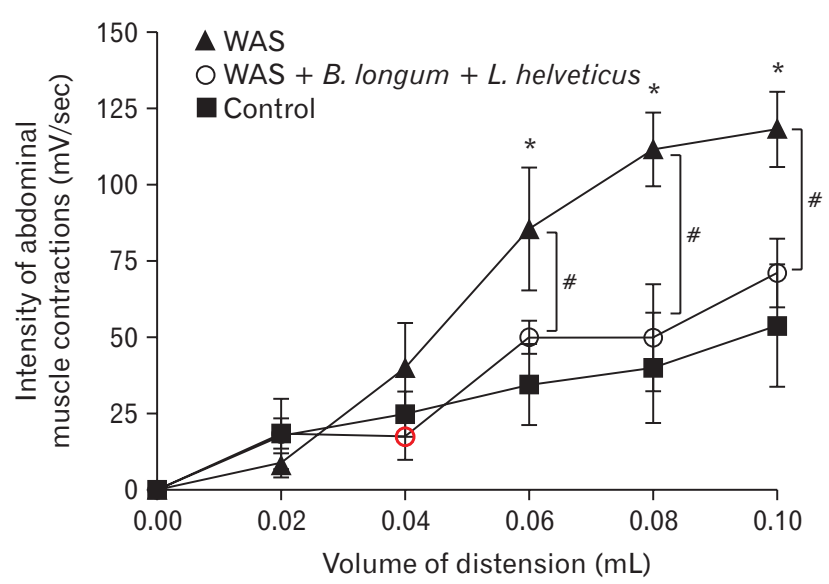

Figure 1. Prevention of abdominal pain induced by chronic stress (water avoidance stress [WAS]) depends on the probiotic strain. ${ }^{*} P$ $<0.05$ and ${ }^{\#} P<0.05$ are significantly different from control and WAS groups. B. longum, Bifidobacterium longum; L. helveticus, Lactobacillus helveticus. Reproduced from original article by AitBelgnaoui et al. ${ }^{1}$ 


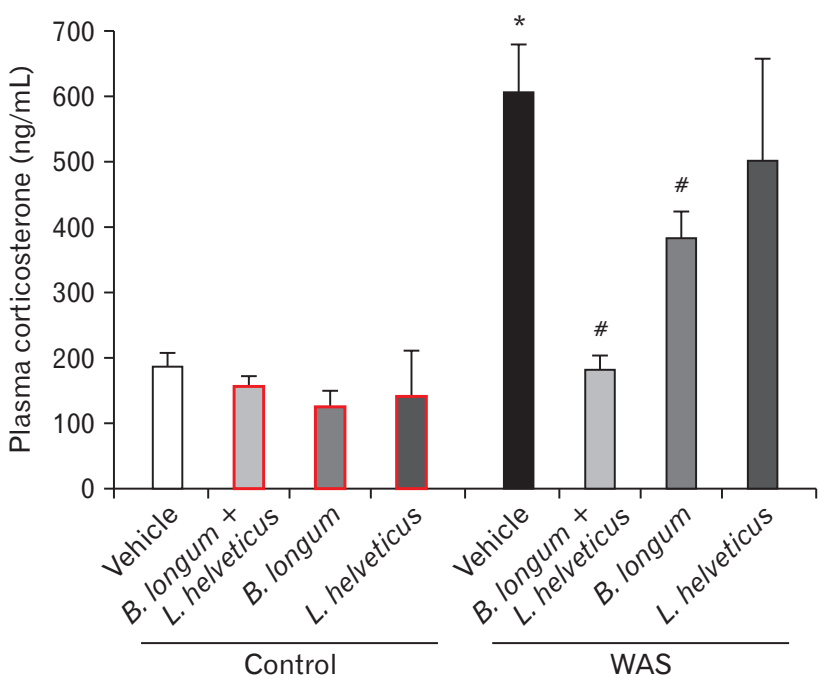

Figure 2. Probiotic treatments differentially prevent a plasma level of stress hormone markers. ${ }^{*} P<0.05$ and ${ }^{\#} P<0.05$ are significantly different from control and water avoidance stress (WAS; chronic stress). B. longum, Bifidobacterium longum; L. helveticus, Lactobacillus helveticus. Reproduced from original article by Ait-Belgnaoui et al. ${ }^{1}$

tion proteins occludin and JAM-A), ${ }^{2}$ and all of these above can be prevented by probiotics.

From our clinician's review, these findings have led to the development of a new understanding of IBS from at least 2 points: (1) there is interrelationship between mental activity, gut symptoms, and the microbiome that is gut-brain-microbiome axis. This axis facilitates bidirectional neural, hormonal, and immunological communication between the gut and brain. In the axis, microbiome serves as an intermediary between the gut and brain, the microbes and their produced peptides and metabolites such as short chain fatty acids can gain access to the areas within the central nervous system and gut, or relay messages to both organs. (2) Chronic stress changes the composition of gut microbiome that initiates IBS and influences its progression and severity, subsequently, dysregulation of this axis produces; or rebalance the composition of gut microbiome by using probiotics can fix the gut-brain-microbiome axis, therefore amelioration of the IBS symptoms. However, these data have not been validated in patients with anxiety and/or depression. In one study on human subjects with depression, no significant difference in the composition of gut microbiota was found between depressed patients and a control group. ${ }^{3}$ Several of the earlier studies have included relatively small and heterogeneous groups of patients, and have not characterized the phenotype of the patients in detail thereby making it impossible to define relevant subgroups. ${ }^{4}$

As marked in red in Figure 1 and 2, it may contain important information but the point seems oversighted in the report. The baselines (control group) of intensity of abdomen muscle contraction and plasma corticosterone level were all remarkably decreased by Bifidobacterium longum and/or Lactobacillus helveticus, especially when normalize the samplings from authors' systematic studies. ${ }^{1,2}$ It postulates that the microbiome play a key role whatever the enteric microbiota in the control group or the WAS group. It may further indicate that the microbiota in IBS pathogenesis could be as an effector, instead of a causer.

In spite that considerable research effort has been forwarded during the last decades, what IBS phenotype is and what is the contribution from microbiota to IBS phenotype or how to maintain or alter such phenotype is still undetermined. We are even not sure the microbiome altered in IBS is the effector or causer.

\section{Anlong Yuan, Peng Li, Li Wang, Bibo Zhong, Bin Bai, Xiaoyu Huang, and Jiguang Kou Department of Internal Medicine, Xiaogan Hospital Affiliated to Wuhan University of Science and Technology, Xiaogan City, Hubei Province, China}

1. Ait-Belgnaoui A, Payard I, Rolland C, et al Bifidobacterium longum and Lactobacillus helveticus synergistically suppress stress-related visceral hypersensitivity through hypothalamic-pituitary-adrenal axis modulation. J Neurogastroenterol Motil 2018;24:138-146.

2. Ait-Belgnaoui, A, Colom, A, Braniste V, el al. Probiotic gut effect prevents the chronic psychological stress-induced brain activity abnormality in mice. Neurogastroenterol Motil 2014;26:510-520.

3. Naseribafrouei A, Hestad K, Avershina E, et al. Correlation between the human fecal microbiota and depression. Neurogastroenterol Motil 2014;26:1155-1162.

4. Simrén M, Barbara G, Flint HJ, et al. Intestinal microbiota in functional bowel disorders: a Rome foundation report. Gut 2013;62:159-176.

Financial support: None. Conflicts of interest: None.

Author contributions: Anlong Yuan, Peng Li, Li Wang, Bibo Zhong, Bin Bai, Xiaoyu Huang, and Jiguang Kou involved in writing and discussion. 